$R M x A C, \mathbf{5 3}, 75-82(2021)$

(c) 2021: Instituto de Astronomía, Universidad Nacional Autónoma de México

https://doi.org/10.22201/ia.14052059p.2021.53.18

\title{
THE BOOTES NETWORK IN THE GRAVITATIONAL WAVE ERA
}

\author{
Y. D. $\mathrm{Hu}^{1,2}$, X. Y. Li ${ }^{1,3}$, A. J. Castro-Tirado ${ }^{1,4}$, E. J. Fernandez-García ${ }^{2}$, A. Castellón ${ }^{5}$, I. M. \\ Carrasco-García $^{6}$, C. J. Perez del Pulgar ${ }^{7}$, M. D. Caballero-García ${ }^{8}$, R. Querel ${ }^{9}$, J. Bai ${ }^{10}$, Y. Fan ${ }^{10}$, S. \\ Guziy $^{11}$, C. Wang ${ }^{10}$, D. Xiong ${ }^{10}$, Y. Xin ${ }^{10}$, X. Zhao ${ }^{10}$, D. Hiriart ${ }^{12}$, W. H. Lee ${ }^{12}$, S. Jeong ${ }^{13}$, and I. H. Park ${ }^{13}$
}

\section{RESUMEN}

BOOTES ("Burst Optical Observer and Transient Exploring System") es una red mundial de telescopios automáticos cuyo objetivo es el seguimiento rápido de fuentes transitorias y astrofísicas en el cielo para el que se instaló la primera estación en 1998. Con la puesta en funcionamiento en 2015 del interferómetro LIGO/Virgo, la nueva generación de detectores diseñados para registrar la señal de ondas gravitacionales, se ha abierto una nueva ventana para explorar el Universo. En esta contribución presentamos el estado de la red BOOTES, la estrategia seguida en el seguimiento de eventos de ondas gravitacionales y sus trabajos en esta Era de Ondas Gravitacionales. También se presentan los resultados preliminares de observaciones.

\section{ABSTRACT}

The Burst Optical Observer and Transient Exploring System (BOOTES) is a world-wide automatic telescope network which aims to repaid follow-up of transient and astrophysical sources in the sky for which the first station was installed in 1998. With the advent in 2015 of the LIGO/Virgo interferometers, as part of the new generation of detectors designed for the detection of the signal of gravitational waves, a new window to explore the Universe has been opened. Here we present the status of the BOOTES network, the related strategies regarding the follow-up of gravitational wave events and the developments in the Gravitational Wave Era. Some preliminary results regarding the BOOTES follow-up observations are presented.

Key Words: gravitational waves — telescopes

\footnotetext{
${ }^{1}$ Instituto de Astrofísica de Andalucía (IAA-CSIC), Glorieta de la Astronomía s/n, E-18008, Granada, Spain (huyoudong072@hotmail.com).

${ }^{2}$ Universidad de Granada, Facultad de Ciencias Campus Fuentenueva S/N CP 18071 Granada, Spain.

${ }^{3}$ National Astronomical Observatories/Nanjing Institute of Astronomical Optics and Technology, Chinese Academy of Sciences, Nanjing 210042, China.

${ }^{4}$ Unidad Asociada CSIC Departamento de Ingeniería de Sistemas y Automática, Escuela de Ingenieros Industriales, Universidad de Málaga, C. Dr. Ortiz Ramos sn, 29071 Málaga, Spain.

${ }^{5}$ Facultad de Ciencias, Universidad de Málaga, Bulevard Louis Pasteur, E-29010 Málaga, Spain.

${ }^{6}$ Departamento de Química Analítica, UMA LASERLAB, Universidad de Málaga, Jimenez Fraud 4, ES 29010, Spain.

${ }^{7}$ Department of Systems Engineering and Automation, Universidad de Málaga, Andalucía Tech, 29071 Málaga, Spain.

${ }^{8}$ Astronomical Institute, Academy of Sciences of the Czech Republic, Boční II 1401, CZ-141 00 Prague, Czech Republic.

${ }^{9}$ National Institute of Water and Atmospheric Research (NIWA), Lauder, New Zealand.

${ }^{10}$ Yunnan Observatories, Chinese Academy of Sciences, 396 Yangfangwang, Guandu District, Kunming, 650216, P.R. China.

${ }^{11}$ Mykolaiv National University, Nikolska 24, Mykolaiv, 54030, Ukraine.

${ }^{12}$ Instituto de Astronomía, Universidad Nacional Autónoma de México, Apartado Postal 70-264, 04510 México, CDMX, México.

${ }^{13}$ Institute for Science and Technology in Space, SungKyunKwan University, Suwon 16419, Republic of Korea.
}

\section{INTRODUCTION AND BACKGROUND}

Massive stars are one of the most interesting targets in the sky, especially their late evolution, which is related to the birth of black hole and neutron stars. As the result of death of massive stars, the resulting compact object will become the ideal natural environment to search for new physical phenomena, related to many types of cataclysmic objects such as supernovae (SN), gamma-ray bursts (GRB), tidal disruptions events (TDE), etc. In the case of a merger of two compact objects, such as a black hole binary (BBH), a neutron star binary (BNS) or a black hole and neutron star binary (BHNS) or even from a super massive star collapse, most of the energy is released through electromagnetic radiation. But a considerable fraction of the angular momentum can be released in the form of gravitational waves $(\mathrm{GW})$ which contains certain information about the progenitors. Hence, the GW signal is a new probe to investigate the nature of the new GW emitter (e.g. initial progenitor masses, the orbit of the binary systen, etc.)

A GW detector was first designed at 1960s by using large cylinders of aluminum that vibrated in response to a passing wave and the sensitivity ap- 
proached $10^{-15} \sim 10^{-17} \mathrm{~m}$. Due to strong noise accompanying the GW signal, the early experiments detected no GW signals. The following generation of GW detectors used the laser interferometry method which monitors the relative motion of freely hanging mirrors. In the $21^{\text {st }}$ century, two km-size interferometers were built and improved the sensitivity up to $10^{-22} \mathrm{~m}$ which made the detection of GW possible. The Laser Interferometer Gravitationalwave Observatory (LIGO), with two stations in the US (Livingston and Hanford) consists of two $4 \mathrm{~km}$ arms. The Virgo interferometer (in Pisa, Italy) consists of two $3 \mathrm{~km}$ arms. During the first scientific observing run, the first GW event was detected on 14 September 2015, which was confirmed as resulting from two $\sim 30$ solar mass black holes merging at a distance of $410_{-180}^{+160} \mathrm{Mpc}$ (Abbott et al. 2016). This historical finding was recognized by awarding Rainer Weiss, Kip Thorne and Barry Barish the Nobel Prize in Physics in 2016 recognizing their earlier work in LIGO.

Near simultaneously to the GW signal, electromagnetic (EM) radiation might reach the Earth and can be used to put constraints on their underlying physical proceses and even on the equation of state of dense nuclear matter (in the case of neutron stars). Therefore, the search for an EM counterpart of a GW source plays a much more important role, especially in the optical band where most ground-based facilities are optimized. As the first finding of GW event with accompanying EM radiation was found in 2017 to be associated with a short-duration GRB (GRB 170817iA; Abbott et al. 2017), this helped to confirm the relation of short-duration GRBs and neutron star binary mergers. As these short-duration GRBs are fast transient events, the rapid localization and prompt follow-up observations are the key to study their properties. Ground-based and spaceborne telescopes are used to perform transient optical observations, while the big-sized telescopes are not suitable for rapid follow-up as they need a precise location of the source and several minutes to repoint the heavy optical tube assemble to the given location on the celestial sphere. On the contrary, smaller robotic telescopes have the advantage of a flexible schedule, quick response and shorter pointing times (seconds only). Besides, robotic telescopes can undertake autonomous operations or even be controlled remotely which makes them easy to be installed worldwide in order to get a better coverage. This is most essential to achieve the continuous monitoring of the transients. Additionally, confirmed optical counterparts at other wavelengths or

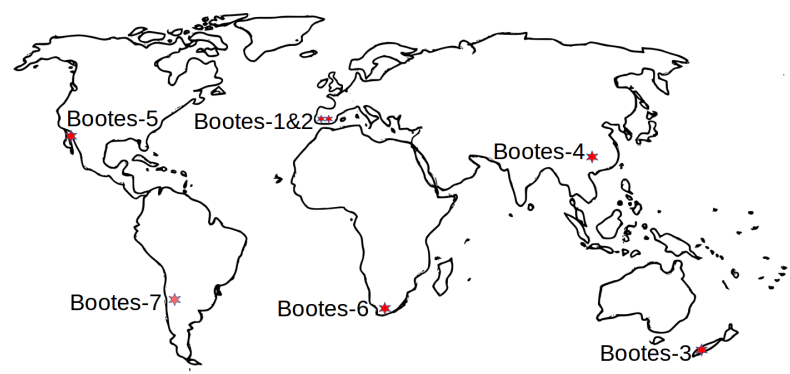

Fig. 1. BOOTES network stations all over the world.

at other observatories can be also followed-up. On this way we can complete the characterization of the GW events by modelling the early prompt emission and their late-time evolution.

\section{BOOTES-NETWORK}

The Burst Optical Observer and Transient Exploring System (BOOTES) network(Castro-Tirado et al. 2012) is a worldwide robotic telescope network which was first designed based on an initial Spanish-Czech collaboration and had first light at 1998. It was the first robotic observatory deployed in Spain and optimized for optical transient followups. But the BOOTES scientific goals are multifold as detailed below.

a) Observations of GRB optical counterparts. Simultaneous optical counterparts to GRBs have been detected in very few cases with white band magnitudes in the range of $\sim 5-10$. These observations provide important results on the central engine and are well suited for BOOTES due to its fast slewing time (few seconds).

b) The detection of optical transients of cosmic origin. These events could be related to new astrophysical phenomena, perhaps associate to Fast Radio Bursts (FRB), neutrinos sources, GWs, Quasars (QSOs), Active Galactic Nuclei (AGN), TDEs, etc.

c) Ground-based support for space missions, such as the ESA International Gamma-Ray Laboratory (INTEGRAL), the Neil Gehrels Swift Observatory (Swift) and the Ultra Fast Flash Observatory (UFFO) (Park et al. 2018) on-board the Lomonosov satellite to monitor high energy targets.

d) Monitoring astronomical galactic objects which include meteors, asteroids, comets, variable stars and novae, etc.

As originally planned, the BOOTES network will consist of 7 stations, four in the northern hemisphere and three in the southern which will ensure that there will always be telescopes that can cover any source either in the northern or in the southern night 
sky. See Figure 1. As of 2020, there are 5 operational observatories so far, which are described below.

The BOOTES-1 observatory is located at the Estación de Sondeos Atmosféricos in the Centro de Experimentación de El Arenosillo which belongs to Institute Nacional de Técnica Aerospacial (CEDEAINTA) in Huelva, Spain (at lat.: $37^{\circ} 5^{\prime} 58.2^{\prime \prime} \mathrm{N}$, long.: $\left.6^{\circ} 44^{\prime} 14.89^{\prime \prime} \mathrm{W}\right)$. The station comprises two domes, BOOTES-1A (B1A) and BOOTES-1B (B1B). B1A is equipped with a wide field CCD camera $(4096 \times 4096$ pix $)$ attached to a $400 \mathrm{~mm} \mathrm{f} / 2.8$ lens which covers a $5^{\circ} \times 5^{\circ}$ field of view (FOV). B1B is a $0.3 \mathrm{~m}$ diameter Schmidt-Cassegrain reflector telescope mounted on a Paramount mount which provides a $15^{\prime} \times 15^{\prime} \mathrm{FOV}$. Any observation with these two devices are obtained in clear filter, which can be calibrated in the R-band, especially in the case of GRB afterglows, under the assumption of no color evolution of the given optical counterparts (Jelínek et al. 2016).

The BOOTES-2/TELMA (B2) observatory is located at Instituto de Hortofruticultura Subtropical y Mediterránea La Mayora which belongs to Consejo Superior de Investigaciones Científicas (IHSM-CSIC) in Algarrobo-Costa, Málaga, Spain (at lat.:36 ${ }^{\circ} 45^{\prime} 24.84^{\prime \prime} \mathrm{N}$, long.: $4^{\circ} 2^{\prime} 33.83^{\prime \prime} \mathrm{W}$ ), $240 \mathrm{~km}$ from the B1 station. This is particularly useful, as we can use simultaneous observations from the two sites in order to discriminate near earth objects using the parallax method. The original $0.3 \mathrm{~m}$ diameter Schmidt-Cassegrain telescope was upgraded to a lightweight $0.6 \mathrm{~m}$ Ritchey-Chretien telescope equipped with fast-slewing mount NTM-500 in 2007. The current CCD camera is a $1024 \times 1024$ pixels EMCCD which provides a FOV of $10^{\prime} \times 10^{\prime}$ equipped with clear, Sloan g; r' i', and WFCAM/VISTA Z and Y filters. In 2012, a light-weight Compact Low Resolution Spectrograph (COLORES; Caballero-García et al. 2014) was installed in B2 covering the wavelength range of $3800-11500 \AA$ with a spectral resolution of $15-60 \AA$ that provides the capability of a preliminary estimation of the redshift of the source in case of the the Lyman-alpha break sitting in the optical band for very-high redshift and very bright optical afterglows.

The BOOTES-3/YA (B3) observatory was first installed at Vintage Lane, Blenheim on 2009, but it was moved to the new location at National Institute of Water and Atmospheric Research (NIWA) in Lauder, Otago, New Zealand (at lat.:45 ${ }^{\circ} 22.92 \mathrm{~S}$, long.: $169^{\circ} 41^{\prime} 0.6^{\prime \prime} \mathrm{E}$ ) staying there since 2014. The BOOTES-4/MET (B4) observatory is located at Lijiang Astronomical Observa- tory which belongs to Yunnan Observatory, Chinese Academy of Sciences, Lijiang, China (at lat.:26 $41^{\prime} 42.8^{\prime \prime} \mathrm{N}$, long.:100¹'48.24"E) since 2012.

The BOOTES-5/JGT (B5) observatory is located at San Pedro Mártir Astronomical Observatory, in Baja California, México (at lat.:31 ${ }^{\circ} 39^{\prime} \mathrm{N}$, long.:115²7’49"E) since 2015.

As a reference, B3, B4 and B5 are all equipped with the same telescope as B2, e.g, a $0.6 \mathrm{~m}$ RitcheyChretien telescope at $\mathrm{f} / 8$ ratio and with an EMCCD covering a field of $10^{\prime} \times 10^{\prime}$ ' with clear, Sloan g; r' i', and WFCAM/VISTA Z and Y filters. The COLORES imaging spectrograph is only available at the B2 station.

The BOOTES-6 (B6) observatory has been partially deployed and it is expected to become operational in 2021. The BOOTES-7 observatory in Chile will become iperational in 2022 .

The BOOTES telescopes reaction time is of the order of 10-20s, thanks to the ultra-light weight optics and a carbon-fiber truss structure. This allows to achieve high slewing speeds $(20-100 \mathrm{deg} / \mathrm{s})$ and accelerations (up to $10 \mathrm{deg} / \mathrm{s}^{2}$ ), so any part of the sky is accessible in less than $8 \mathrm{~s}$.

Besides, each station hosts an all-sky camera (named Compact All-Sky Automated Network Developed for Research in Astronomy, CASANDRA). This is a $4096 \times 4096$ pixels $^{2}$ CCD attached to a $16 \mathrm{~mm} \mathrm{f} / 2.8$ lens that covers a FOV of $180^{\circ}$. The resulting images are recorded every minute showing the sky conditions (clouds, etc.) and detecting stars brighter than 10 mag.

\section{LIGO/VIRGO OBSERVING RUNS}

The LIGO/Virgo GW detectors have already completed three scientific observing runs. The first observing run (O1) was conducted by the advanced LIGO detectors in Hanford and Livingston, starting on September $12^{\text {th }}, 2015$ and ending on January $19^{\text {th }}$ 2016. After ten months of an upgrading and commissioning period, the second observing run (O2) took place between 30 Nov 2016 and 25 Aug 2017, which initially was executed by the LIGO detector. The advanced Virgo detector joined the running on 1 August 2017, enabling the first three-detector observations of GWs. On 1 Apr 2019, LIGO and Virgo observatories were devoted during a period of 19 months to further engineering and commissioning works, prior to the start of the third observing run (O3), which took place for one year long until April 2020. During the first two runs, the positions of the GW alerts on the sky were distributed only to those teams which signed memorandums of under- 




Fig. 2. BOOTES strategy sketch map in the O3 period.

standing (MoU) with the LIGO/Virgo Collaboration while these positions became public for O3.

\section{BOOTES FOLLOW-UP STRATEGY AND OBSERVING METHODS FOR O3}

In previous observing runs, the localization of the GW events was reported with huge errors which covered several thousands $d e g^{2}$. The best located event so far was GW 170817, thanks to the joint Virgo detection. The GW position could be initially nailed down to to $28 \mathrm{deg}^{2}$ and then refined to $16 \mathrm{deg}^{2}$, still a considerable large region to survey. Hence a optimized method in O3 was needed to cover those regions as soon as possible aiming at the early discovery of the corresponding candidates.

The BOOTES network, comprising of wide-field and narrow-field telescopes, can efficciently make use of them in order to search for an optical counterpart. The wide-field camera is used to tile the sky localization probability maps with aim of covering as much of $2 \mathrm{D}$ probability localization as possible. The narrow-field telescope, targeting specific galaxies in the 3D sky region will significantly improve upon the depth of the wide-field cover for those galaxies hosting the GW event. Besides, the simultaneous GRB trigger is set to the highest priority in case there is another GW event similar to GW 170817 which can be precisely located by a satellite. The specific strategy is implemented as follows.

The GW alerts sent through the Gamma-ray Coordinates Network (GCN) are of two types: human- readable GCN circulars and machine-readable GCN Notices. We use the type of GCN notices in the VO-Event XML format. After receiving an alert, a type identification module will analyzes the notice contents to distinguish four kinds of GCN Notices: Preliminary, Initial, Update and Retraction. If of non-retraction type, it gets the all-sky image stored in the HEALPix (Górski et al. 2005) projection and the value stored at each pixel is the probability that the GW source is within that pixel. Since B1A is a $5^{\circ} \times 5^{\circ}$ FOV telescope, it can scan the $90 \%$ of the sky for the events in one night. The algorithm mosaic divides all the sky into mosaics of $5^{\circ} \times 5^{\circ}$ and integrates the probabilities in each mosaic. The rank by the probabilities for the observation of B1A can be got from the center coordinates of the mosaics. Based on the powerful BOOTES robotic observing system, the observations can be submitted programmatically. All we need is just creating an URL to take all the parameters needed, including priorities, exposure time, etc, and send them through wget command to the telescope website. Four standardized BOOTES telescopes, from B2 to B5, with $10^{\prime} \times 10^{\prime}$ FOV and B-1B with $15^{\prime} \times 15^{\prime}$ FOV are used for observing galaxies. The event identification gets the probability that the source is a BNS or a NSBH. If one of them is larger than 0.5 , the galaxies scheduling algorithms determines the pointing pattern of the telescopes by covering the regions where the source is most probably located at, then select galaxies from GLADE database that match the distance from the 


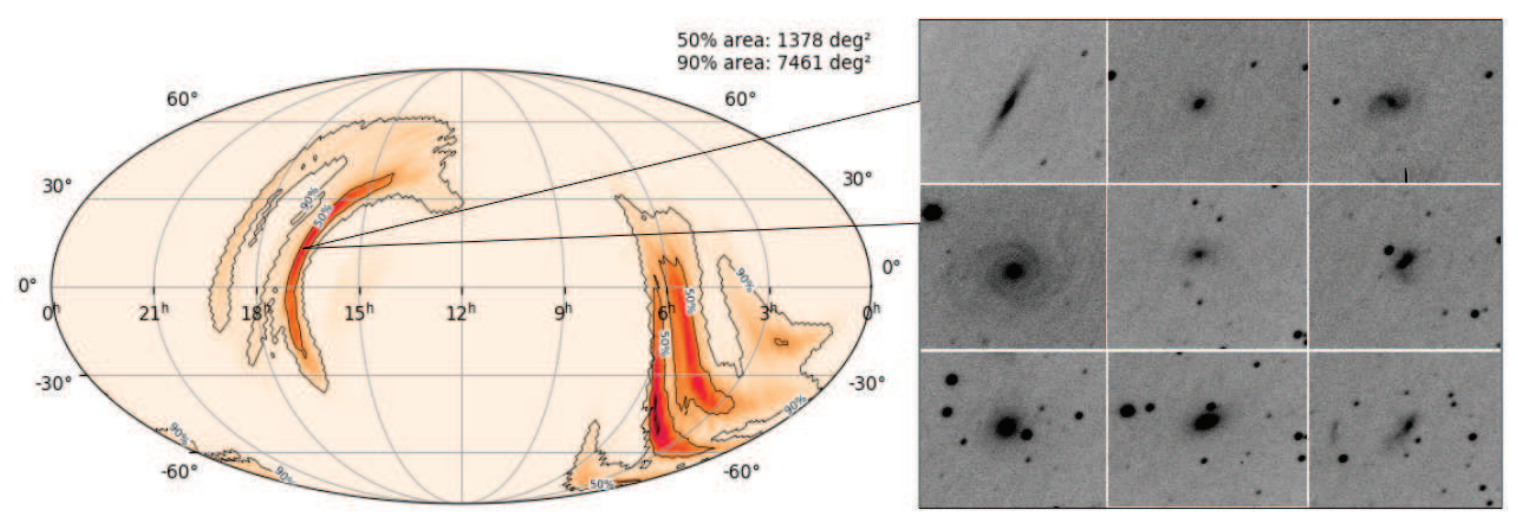

Fig. 3. Example images of BOOTES galaxies targeting from BOOTES-5 for the event GW 190425.

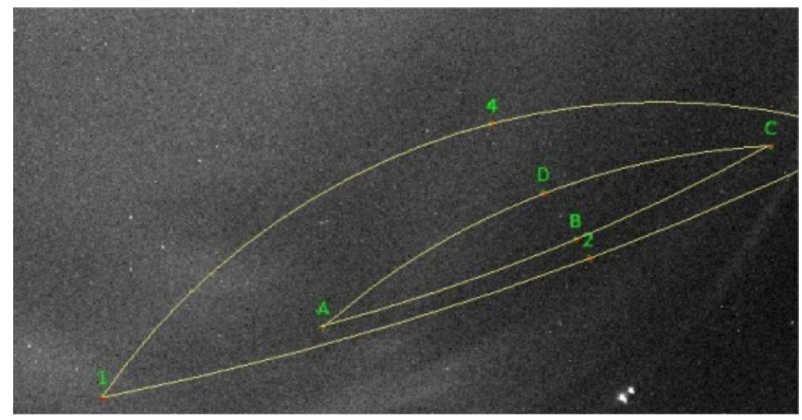

Fig. 4. CASANDRA-3 all sky camera simultaneous image from BOOTES-3 database for GW 150914. The region (A-B-C-D, 1-2-3-4) represents the $50 \%$ and $90 \%$ error region from the LIGO/Virgo probability map.

region. The coordinates of the galaxies are written into a file with the format that BOOTES telescope's website can recognize. The standard B-1A exposure per frame is $300 \mathrm{sec}$ and for other telescopes $20 \mathrm{sec}$, so that the typical number of observed frames per night is $\sim 75$ for $\mathrm{B}-1 \mathrm{~A}$ and $\sim 1000$ for the rest of the telescopes. Hence, a cut is employed when the GW uncertainty is large in order to limit the number of targeted galaxies to less than 1000 per night. The algorithm details the specific process is shown in Figure2.

\section{BOOTES OBSERVATIONAL RESULTS IN O1 AND O2}

In the past two observing runs (O1 and $\mathrm{O} 2$ ), there have been 11 confirmed GW events observed by LIGO/Virgo including BBH, BHNS and BNS (Abbott et al. 2019). We could make use of the BOOTES network archival data in order to search and follow up some particular events.

\section{1. $G W 150914$}

On 14 Sep 2015 at 09:50:45 UT the first direct detection of an astrophysical gravitational wave sig-

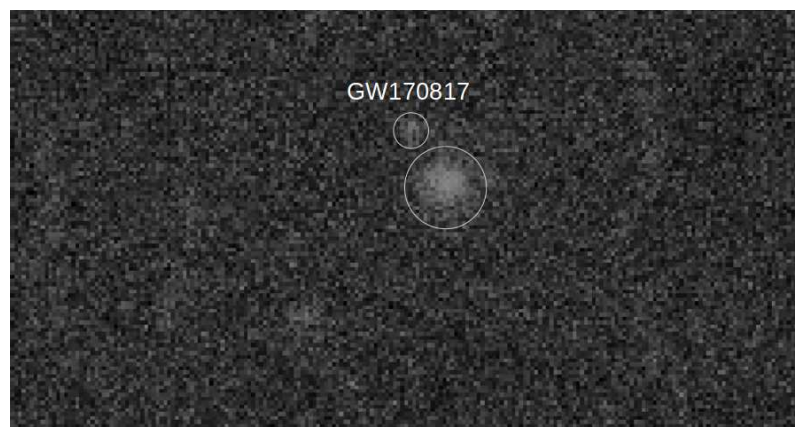

Fig. 5. BOOTES-5/JGT observation for GW 170817 event. The small circle is the source position and the bigger circle represent its host galaxy NGC4993.

nal was recorded during the O1 run. It was detected simultaneously by the two LIGO detectors and the signal turned out to result from a binary black hole merger at a galaxy with a redshift of $\mathrm{z}=0.09_{-0.04}^{+0.03}$. It was initially dubbed G184098 and later it was given its name following the date of the wavefront arrival to Earth: GW 150914 (Abbott et al. 2016).

For this particular event, the CASANDRA-3 all sky camera at BOOTES-3 station covered the entire southern sky, including the hugh error box for GW 150914. This was the only simultaneous observations ever taken for the first GW alert. Searching for the bright optical transient was conducted in B3 archival data. No prompt optical emission was found and the image reached a limiting magnitude of 5 due to poor weather conditions (Castro-Tirado et al. 2016; Abbott et al. 2016). See Figure 4.

\section{2. $G W 170817$}

On 17 Aug 2017 at 12:41:04 UT, the advanced LIGO and Virgo detectors detected the merger of a binary neutron star system during the $\mathrm{O} 2$ run. Meanwhile the Fermi Gamma-ray Burst Monitor independently detected a short gamma-ray burst 
(GRB 170817A) with a time delay of $1.7 \mathrm{~s}$ with respect to the merger time set by the GW detection. A careful analysis revealed the two events were associated and related to GW 170817. The modelling of the GW signal implied that a binary neutron star system with a mass of the two components of $2.74_{0.01}^{+0.04} \mathrm{M}_{\text {sun }}$ merged at a luminosity distance of $40_{-8}^{+8} \mathrm{Mpc}$, producing the resulting $\mathrm{GW}$ emission. It was the first direct evidence of the link of binary neutron stars mergers and short gamma-ray bursts (Abbott et al. 2017).

The JGT telescope at the BOOTES-5 station started imaging 15 galaxies which were selected by cross coincidence of the GLADE catalogue and the GW 170817 localization probability map on Aug 18.21 UT. We detected the optical counterpart reported by other teams (SSS17a) to be located in the outskirts of its host galaxy, NGC 4993, with a magnitude $\mathrm{r}=18.20 \pm 0.45$ in the B5 coadded images, a value which is consistent with other contemporaneous measurements (Zhang et al. 2018).

\section{BOOTES OBSERVATIONS IN O3}

From the lessons learned in the $\mathrm{O} 1$ and $\mathrm{O} 2$ runs, any GW trigger detected by LIGO/Virgo had to be related to a compact binary coalescence $(\mathrm{CBC})$ but due to the increased sensitivity in O3, specific bursts resulting from a sigle compact object collapse could be expected. The nature of the former one (BNS, $\mathrm{BBH}, \mathrm{NSBH}$ and Mass Gap) can be inferred from the modelling of the signal whereas for the second one, spikes with a duration of $\sim 1 \mathrm{sec}$ and for which its origin is still not known, could be spotted. Since the start of the $\mathrm{O} 3 \mathrm{run}, 37$ triggers were recorded (all CBC events) until 30 Sep 2019. They include 21 BBHs mergers, 10 BNS mergers, 2 Mass gap events and 4 events whose astrophysical origin.

The BOOTES network has responded to most of GW alerts since the beginning of $\mathrm{O} 3$ and followed up 31 events of them, which include 19 BBHs, 9 NSrelated mergers, 1 Mass gap event and 2 retracted events that represent $84 \%$ of all triggers. The regions tiled by $\mathrm{B} 1 \mathrm{~A}$ and the targeted galaxies imaged by other BOOTES telescopes are listed in Table 1.

Hereafter, we select GW 190425 as an example to briefly show the results of our search. A sketch of our selected galaxies is shown in Figure 3, considering the probability map provided for GW 190425 .

\section{1. $S 190425 z$}

On 25 April 2019 at 08:18:05 UT, the LIGO detector at Livingston observed a new BNS merger event while the detector at Hanford was offline. The
TABLE 1

TABLE OF BOOTES GW OBSERVATIONS UNTIL 30 SEP 2019

\begin{tabular}{|c|c|c|c|c|c|c|c|}
\hline \multirow[b]{2}{*}{ Name } & \multirow[b]{2}{*}{ Type* } & \multicolumn{3}{|l|}{ Region } & \multicolumn{2}{|c|}{ Galaxy } & \multirow[b]{2}{*}{ B5 } \\
\hline & & B1A & B1B & B2 & B3 & B4 & \\
\hline S190408an & $\mathrm{CBC}(\mathrm{a})$ & $37 / 43$ & 0 & 0 & 0 & 0 & 0 \\
\hline S190412m & $\mathrm{CBC}(\mathrm{a})$ & $25 / 25$ & 0 & 0 & 0 & 0 & 0 \\
\hline S190421ar & $\mathrm{CBC}(\mathrm{a})$ & $52 / 88$ & 0 & 0 & 0 & 0 & 0 \\
\hline S190425z & $\mathrm{CBC}(\mathrm{d})$ & $1 / 280$ & 0 & 1 & 0 & 165 & 63 \\
\hline S190426c & $\mathrm{CBC}(\mathrm{c} / \mathrm{d})$ & $48 / 106$ & 241 & 615 & 615 & 273 & 501 \\
\hline S190503bf & $\mathrm{CBC}(\mathrm{a})$ & $1 / 78$ & 0 & & 0 & 0 & 0 \\
\hline S190510g & $\mathrm{CBC}(\mathrm{c} / \mathrm{d})$ & $10 / 135$ & 0 & 50 & 27 & 31 & 155 \\
\hline S190512at & $\mathrm{CBC}(\mathrm{a})$ & $0 / 40$ & 69 & 52 & 800 & 356 & 373 \\
\hline S190513bm & CBC(a) & $0 / 61$ & 18 & 0 & 0 & 0 & 595 \\
\hline S190517h & $\mathrm{CBC}(\mathrm{a})$ & $0 / 75$ & 0 & 0 & 0 & 54 & 0 \\
\hline S190519bj & $\mathrm{CBC}(\mathrm{a})$ & $0 / 70$ & 0 & 0 & 1018 & 83 & 0 \\
\hline S190521g & CBC(a) & $0 / 79$ & 38 & 0 & 0 & 0 & 4 \\
\hline S190521r & $\mathrm{CBC}(\mathrm{a})$ & $0 / 53$ & 10 & 0 & 2 & 1 & 0 \\
\hline S190602aq & $\mathrm{CBC}(\mathrm{a})$ & $0 / 79$ & 0 & 0 & 272 & 0 & 19 \\
\hline S190630ag & $\mathrm{CBC}(\mathrm{a})$ & $14 / 246$ & 0 & 0 & 0 & 0 & 0 \\
\hline S190701ah & $\mathrm{CBC}(\mathrm{a})$ & $4 / 13$ & 0 & 0 & 0 & 0 & 25 \\
\hline S190706ai & $\mathrm{CBC}(\mathrm{a})$ & $17 / 73$ & 192 & 0 & 0 & 0 & 0 \\
\hline S190707q & $\mathrm{CBC}(\mathrm{a})$ & $37 / 108$ & 1 & 1 & 0 & 0 & 0 \\
\hline S190718y & $\mathrm{CBC}(\mathrm{c} / \mathrm{d})$ & $4 / 66$ & 98 & 319 & 0 & 0 & 0 \\
\hline S190720a & $\mathrm{CBC}(\mathrm{a})$ & $49 / 108$ & 226 & 163 & 0 & 0 & 0 \\
\hline S190727h & $\mathrm{CBC}(\mathrm{a})$ & $25 / 92$ & 0 & 474 & 0 & 0 & 0 \\
\hline S190728q & $\mathrm{CBC}(\mathrm{a})$ & $13 / 79$ & 614 & 951 & 0 & 0 & 0 \\
\hline S190808ae & $\mathrm{CBC}(\mathrm{c} / \mathrm{d})$ & $20 / 176$ & 0 & 240 & 1 & 0 & 0 \\
\hline S190814bv & $\mathrm{CBC}(\mathrm{d} / \mathrm{b})$ & $0 / 73$ & 0 & 103 & 17 & 0 & 296 \\
\hline S190816i & $\mathrm{CBC}(\mathrm{b})$ & $0 / 85$ & 0 & 0 & 0 & 0 & 0 \\
\hline S190822c & $\mathrm{CBC}(\mathrm{d})$ & $0 / 112$ & 10 & 0 & 0 & 0 & 352 \\
\hline S190828j & $\mathrm{CBC}(\mathrm{a})$ & $0 / 54$ & 0 & 0 & 0 & 0 & 0 \\
\hline S190828l & $\mathrm{CBC}(\mathrm{a})$ & $0 / 68$ & 0 & 0 & 0 & 0 & 0 \\
\hline S190829u & $\mathrm{CBC}(\mathrm{e} / \mathrm{c})$ & $0 / 315$ & 0 & 0 & 0 & 0 & 0 \\
\hline S190901ap & $\mathrm{CBC}(\mathrm{d})$ & $0 / 311$ & 0 & 324 & 0 & 0 & 0 \\
\hline S190910d & $\mathrm{CBC}(\mathrm{b})$ & $2 / 155$ & 18 & 21 & 0 & 0 & 448 \\
\hline S190910h & $\mathrm{CBC}(\mathrm{d} / \mathrm{c})$ & $22 / 268$ & 0 & 0 & 0 & 0 & 0 \\
\hline S190915ak & $\mathrm{CBC}(\mathrm{a})$ & $2 / 40$ & 8 & 0 & 0 & 0 & 253 \\
\hline S190923y & $\mathrm{CBC}(\mathrm{b} / \mathrm{c})$ & $0 / 113$ & 0 & 0 & 0 & 0 & 0 \\
\hline S190924h & $\mathrm{CBC}(\mathrm{e})$ & $0 / 49$ & 0 & 51 & 0 & 0 & 0 \\
\hline S190930s & $\mathrm{CBC}(\mathrm{e})$ & 0 & 0 & 0 & 0 & 0 & 0 \\
\hline S190930t & $\mathrm{CBC}(\mathrm{b} / \mathrm{c})$ & $2 / 275$ & 4 & 50 & 0 & 0 & 0 \\
\hline
\end{tabular}

"a:BBH b:NSBH c:Terrestrial d:BNS e:Mass gap.

Virgo detector took data but could not contribute to improve the localisation because of its low signal-tonoise ratio (Ligo Scientific Collaboration \& VIRGO Collaboration 2019). Hence, this was the first BNS event in O3, which was reported with the $90 \%$ prob- 
TABLE 2

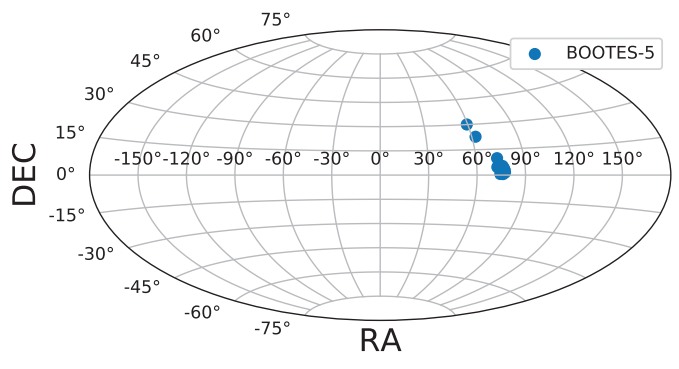

Fig. 6. BOOTES-5 galaxies observation coverage for the GW 190425.

ability map covering $10183 \mathrm{deg}^{2}$, an area which was further reduced to $7461 \mathrm{deg}^{2}$. Neither satellite nor neutrino detector found any coincidence with this GW signal at the same time. It was reported that this event originated from a rare binary neutron star system with total mass $3.4_{-0.1}^{+0.3} \mathrm{M}_{\text {sun }}$. Since its mass is significantly large compared to the population of other known BNS systems it was to be one rare event happening every 69000 years. The system distance was estimated to be $156 \pm 41 \mathrm{Mpc}$ (The LIGO Scientific Collaboration et al. 2020).

As in other events, the BOOTES network followed the standard process, where submitted galaxy targets are selected from the GLADE catalogue within the give $\mathrm{GW}$ error area visible at a given BOOTES location. Thus the BOOTES-5/JGT telescope observed 63 galaxies within the GW 190425 uncertainty region (as shown in Figure 6, which list the coverage of the galaxies observations) starting at 9:57:38 UT. Furthermore, the BOOTES-4/MET telescope observed 165 galaxies during the night of April $25^{\text {th }}$. For each galaxy, $60 \mathrm{~s}$ exposure in the clear filter were obtained, with the limiting magnitude affected by the poor weather conditions. All those images were reduced with the standard method used with IRAF package and compared with the reference image taken from the same region of the sky in both the USNO-B1.0 and the Pan-STARRS catalogs. No new transient sources were found in the data analysis of those fields. Using these measurements, we reported the typical 3- $\sigma$ upper limit of 20.5 mag for the BOOTES-5 frames (Hu et al. 2019).

\section{CONCLUSION}

We have presented the BOOTES network status nowadays and its strategy optimized for GW optical counterpart searches, which includes tiling for covering the GW localization probability map

GALAXIES OBSERVED BY B5 FOR GW 190425

\begin{tabular}{|c|c|c|c|c|}
\hline & $\begin{array}{c}\mathrm{RA} \\
\text { (Deg) }\end{array}$ & $\begin{array}{c}\text { DEC } \\
\text { (Deg) }\end{array}$ & & $\begin{array}{c}\delta \mathrm{T} \\
(\mathrm{hrs})\end{array}$ \\
\hline & 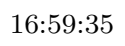 & & & 1.66 \\
\hline & & & & 79 \\
\hline & 0 & & & 1.81 \\
\hline & $10:$ & & & .86 \\
\hline & 9 & & & 91 \\
\hline & $16: 5$ & & Clear & 2.04 \\
\hline & $16: 5$ & & & .07 \\
\hline & 26 & & ar & 10 \\
\hline & & & Clear & 2.12 \\
\hline & & & & 14 \\
\hline & $17: 0$ & & ear & 2.17 \\
\hline & & & $\operatorname{ar}$ & 2.20 \\
\hline & & & & 22 \\
\hline & $17:($ & & Clear & 2.25 \\
\hline & & & & 27 \\
\hline & & & & 2.29 \\
\hline & 17: & & Clear & 2.32 \\
\hline & & & & .34 \\
\hline & & & & 2.36 \\
\hline & & & & 39 \\
\hline & & & & 41 \\
\hline & & & $\mathrm{Cl}$ & 2.44 \\
\hline & 17: & & & 47 \\
\hline & & & & 2.49 \\
\hline & & & $\mathrm{Cl}$ & 2.51 \\
\hline & & & & 54 \\
\hline & & & & 2.56 \\
\hline & & & & .59 \\
\hline & & & ar & 2.61 \\
\hline & & & & 2.63 \\
\hline & & & & 2.66 \\
\hline & & & & 2.68 \\
\hline & & & & 2.71 \\
\hline & 37 & 13 & $\operatorname{ar}$ & .73 \\
\hline & & & & 2.75 \\
\hline & & & & 2.78 \\
\hline & 16 & & ar & 2.80 \\
\hline & & & Clear & 2.82 \\
\hline & & & & 2.85 \\
\hline & & & & 2.87 \\
\hline & & & & 2.90 \\
\hline & 16: & +0 & $\operatorname{ar}$ & 2.92 \\
\hline 045 & $16:$ & & Clear & 2.94 \\
\hline & & & & 2.97 \\
\hline & & + & & 2.99 \\
\hline 0202344 & $17: 01: 42$ & & Clear & 3.02 \\
\hline & & & & 3.04 \\
\hline 81 & $16: 55: 06$ & $+03:$ & Clear & 3.06 \\
\hline & & & & 3.09 \\
\hline & & & & 3.1 \\
\hline $2 \mathrm{MASS} 16541350+0$ & $16: 54: 13$ & $+05: 03: 28$ & Clear & 3.14 \\
\hline
\end{tabular}


TABLE 2. CONTINUED

\begin{tabular}{ccccc}
\hline Galaxy & $\begin{array}{c}\text { RA } \\
(\mathrm{Deg})\end{array}$ & $\begin{array}{c}\text { DEC } \\
(\mathrm{Deg})\end{array}$ & $\begin{array}{c}\text { Band } \\
(\mathrm{hrs})\end{array}$ \\
\hline 2MASS16521538+0343210 & $16: 52: 15$ & $+03: 45: 22$ & Clear & 3.16 \\
2MASS16521412+0314088 & $16: 52: 14$ & $+03: 16: 10$ & Clear & 3.18 \\
2MASS16514686+0334557 & $16: 51: 47$ & $+03: 36: 57$ & Clear & 3.93 \\
2MASS16504997+0437178 & $16: 50: 50$ & $+04: 39: 19$ & Clear & 3.95 \\
2MASS16503422+0505350 & $16: 50: 34$ & $+05: 07: 36$ & Clear & 3.98 \\
2MASS16494530+0416395 & $16: 49: 45$ & $+04: 18: 41$ & Clear & 4.00 \\
2MASS16503128+0209319 & $16: 50: 52$ & $+02: 11: 33$ & Clear & 4.02 \\
UGC10104 & $15: 57: 28$ & $+30: 05: 37$ & Clear & 4.05 \\
HyperLEDA1843124 & $15: 47: 24$ & $+28: 43: 32$ & Clear & 4.07 \\
2MASS15585202+2608083 & $15: 58: 52$ & $+26: 10: 09$ & Clear & 4.10 \\
2MASS15562377+2549058 & $15: 56: 23$ & $+25: 51: 07$ & Clear & 4.12 \\
SDSSJ160952.75+223818.6 & $16: 09: 53$ & $+22: 38: 19$ & Clear & 4.15 \\
\hline
\end{tabular}

for the BOOTES wide field cameras and targeting galaxies observations for the narrow field telescopes. These strategies work properly since the start of O3 and BOOTES network followed-up $84 \%$ of the alerts from LIGO/Virgo. Although no EM candidates were found for any GW alert such as GW 190425, BOOTES observations excluded an optical counterpart brighter than 20.5 mag lying in the vecinity of the observed galaxies.

With the O3 run ending on 30 April 2020 and the next observing run O4starting in 2022, we expect all the remaining BOOTES stations will become operational by that time, in order to continue playing an important role in the searching of GW optical counterparts.
Acknowledgements: We acknowledge support from the Spanish MINECO ministry and European FEDER funds AYA-2015-71718-R. This work has been partially supported by the Spanish Science Ministry "Centro de Excelencia Severo Ochoa" Program under grant SEV-2017-0709.

\section{REFERENCES}

Abbott, B. P., Abbott, R., Abbott, T. D., et al. 2016, ApJS, 225, 8

$$
\begin{aligned}
& \text { 2016, PhRvL, 116, } 1102 \\
& \text { 2017, PhRvL, 119, } 161101 \\
& \text { 2017, ApJ, 848, 12 } \\
& \text { 2019, PhRvX, 9, } 1040 \\
& \text { 2020, ApJ, 892, 3 }
\end{aligned}
$$

Caballero-García, M. D., Jelinek, M., Castro-Tirado, A., et al. 2014, arXiv e-prints, arXiv:1408.4370

Castro-Tirado, A. J., Jelínek, M., Gorosabel, J., et al. 2012, ASInC, 7, 313

Castro-Tirado, A. J., Tello, J. C., Cunniffe, R., et al. 2016, GCN, 19022, 1

Castro-Tirado, A. J., Tello, J. C., Hu, Y., et al. 2017, GCN, 21624, 1

Górski, K. M., Hivon, E., Banday, A. J., et al. 2005, ApJ, 622,759

Hu, Y.-D., Li, X.-Y., Carrasco, I., et al. 2019, GCN, 24270, 1

Jelínek, M., Castro-Tirado, A. J., Cunniffe, R., et al. 2016, AdAst, 2016, 12

Park, I. H., Panasyuk, M. I., Reglero, V., et al. 2018, SSRv, 214, 14

The LIGO Scientific Collaboration, \& VIRGO Collaboration. 2019, GCN, 24168, 1

Zhang, B.-B., Zhang, B., Sun, H., et al. 2018, NatCo, 9, 447 